\title{
Ethnologies
}

\section{La migration transnationale d'un patrimoine vivant}

Le cas de la fête du Ngondo des peuples sawa célébrée à

Montréal et à Douala

\section{Robinson Ngametche}

Volume 34, numéro 1-2, 2012

URI : https://id.erudit.org/iderudit/1026155ar

DOI : https://doi.org/10.7202/1026155ar

Aller au sommaire du numéro

Éditeur(s)

Association Canadienne d'Ethnologie et de Folklore

ISSN

1481-5974 (imprimé)

1708-0401 (numérique)

Découvrir la revue

Citer cet article

Ngametche, R. (2012). La migration transnationale d'un patrimoine vivant : le cas de la fête du Ngondo des peuples sawa célébrée à Montréal et à Douala.

Ethnologies, 34(1-2), 301-318. https://doi.org/10.7202/1026155ar d'utilisation que vous pouvez consulter en ligne.

https://apropos.erudit.org/fr/usagers/politique-dutilisation/ 


\section{LA MIGRATION TRANSNATIONALE D'UN PATRIMOINE VIVANT}

Le cas de la fête du Ngondo des peuples sawa célébrée à Montréal et à Douala

\section{Robinson Ngametche}

Université Laval

\section{Introduction}

Le festival Ngondo Afro-Monde de Montréal est la représentation de la fête traditionnelle camerounaise dénommée Ngondo, célébrée par les peuples sawa ${ }^{1}$ au bord du fleuve Wouri à Douala pendant la période de fin d'année. Cette fête voit le jour à Montréal pour la première fois en 2009 par l'événement « Ngondo ô Canada 2009 », sous la coordination du président du Ngondo, sa majesté le prince René Douala Manga Bell. Cette fête se caractérisant par des rituels et des processions liés à l'environnement, le Ngondo attire la curiosité d'un très grand public. L'étude de la fête du Ngondo au Cameroun et au Canada me semble importante afin de pouvoir établir une comparaison à travers notamment les différences, le changement ou les transformations qu'elle a connues, compte tenu des nouvelles réalités dans un pays d'accueil.

Le Ngondo est l'Assemblée traditionnelle et la fête annuelle des Sawa du Cameroun, et sa mission est d'invoquer les esprits des ancêtres, de régler les litiges et les tensions entre les différents clans, aussi mystiques soient-ils, pour trouver des solutions appropriées et consensuelles aux problèmes que rencontrent les populations, ceci par le biais des rituels et des processions destinés à améliorer les conditions de vie des peuples (Doumbé Moulongo 1972). Le Ngondo, à l'origine, était considéré comme une instance juridictionnelle prenant des décisions exécutoires ; il représentait un organe de régulation des termes d'échanges ; il était en outre le moyen imparable

1. Sawa est la désignation des peuples autochtones riverains de l'océan Atlantique, du sud-ouest et d'une partie du sud du Cameroun. Ce terme signifie aussi « côtier ». 
par lequel tout Sawa pouvait comprendre le monde et son environnement. À cet effet, le Ngondo représentait l'alpha et l'oméga de toute personne faisant partie de la lignée sawa (Élamé 2006). Esoh Élamé, dans son étude, va plus loin en soutenant que le Ngondo en tant que forme de religion traditionnelle africaine, est omniprésent dans la vie quotidienne car il incarne la culture chez les peuples sawa. Il ne constitue pas seulement le moment des festivités. C'est un processus accompagnant chaque Sawa de la naissance à la mort et son contenu est précieux pour l'édification de l'identité de l'individu (Élamé 2006).

Le Ngondo apparaît au demeurant à la fois comme un mode de vie et comme une fête traditionnelle très significative pour les peuples sawa. Ses festivités ont lieu généralement vers la fin de l'année, soit la première semaine du mois de décembre ; elles commencent à partir de l'annonce officielle par les chefs Sawa du thème retenu cette année-là et de la période devant couvrir les événements. Il se caractérise par un défilé carnavalesque dans les rues de Douala, le dépôt de gerbes de fleurs sur les tombes des héros ${ }^{2}$ sawa, des rituels tels que la messe de l'eau où on assiste à l'immersion du vase sacré $^{3}$ dans le fleuve Wouri ${ }^{4}$, l'exposition d'objets d'art, enfin son apothéose qui se caractérise par la liesse populaire lors de multiples jeux et concours (Épée 2006). Le déplacement d'une délégation camerounaise pour présider à cette fête au Canada ne pouvait que susciter beaucoup d'émotion, tant au Cameroun qu'ici au Canada.

2. Ceux qui ont marqué l'histoire de la communauté par un acte spécial, par exemple le roi Rodolphe Douala Manga Bell, exécuté par pendaison en 1914 par les Allemands, pour avoir osé revendiquer les droits de son peuple au sujet du problème de l'expropriation des terre à Douala par l'administration coloniale allemande (MVENG 1963).

3. L'immersion du vase sacré est un rituel typiquement sawa, ayant une charge mystique extrêmement forte ; lors de ce rituel, un initié muni d'un vase relativement volumineux plonge au fond du fleuve Wouri et en ressort au bout d'une demi-heure environ sans la moindre goutte d'eau sur son vêtement ni sur le vase qu'il tient ; dans le vase se trouve un « Digombo », un crabe beaucoup plus agressif qu'un crabe ordinaire, et qui représente un message venant des ancêtres sawa et dont le destinataire est le peuple. Ce message est décrypté et interprété par les seuls initiés et dignitaires sawa ; il renferme le plus souvent des prédictions au sujet de l'ensemble des activités des Sawa pour l'année à venir ; cela peut porter sur les récoltes, la fécondité des femmes, les activités de pêche, mais il peut renfermer aussi des mises en garde contre certaines difficultés pouvant advenir dans la population (Doumbe Moulongo 1972).

4. Fleuve qui se jette dans l'océan Atlantique et qui abrite le principal port du Cameroun. 
Pourquoi doit-on pratiquer une fête traditionnelle comme le Ngondo hors de son contexte d'origine ? Pourquoi doit-on déplacer le Ngondo ? Estce qu'un Ngondo à l'étranger n'est pas la profanation des valeurs ancestrales sur lesquelles se base toute la mémoire même de cette fête ? N'est-ce pas une transformation totale de la fête ? Répondre à ces interrogations constitue le fond de cet article. Ma recherche vise à mieux comprendre comment la fête du Ngondo à Montréal prend un sens tout à fait différent de celui qu'elle a au Cameroun, selon les contextes et les situations de pratiques qui varient d'un lieu à un autre. Il est question de comprendre le processus de changement ou d'évolution de cette fête, comprendre le sens que donnent les Sawa eux-mêmes à cette fête dans un pays d'accueil.

Plusieurs études m'ont inspiré et m'ont permis de comprendre et de préciser l'objet de la recherche, en l'occurrence l'étude de Linda Guidroux qui éclaire les formes d'appartenance culturelle de la communauté bretonne en situation de mobilité géographique. L'auteure étudie, à travers sa thèse de doctorat, comment l'appartenance culturelle régionale bretonne a été déterritorialisée, puis relocalisée au Québec. Elle considère donc la migration comme une situation expérimentale à travers laquelle une appartenance culturelle peut se déconstruire ou se reconstruire. Cette étude m'a permis de comprendre comment les Sawa du Canada manifestent eux aussi certains aspects de leur appartenance culturelle (Guidroux 2011). Dans la même veine, les travaux de Séverine Dessajan, par sa thèse de doctorat «Les Duala et le Ngondo, ou comment une assemblée traditionnelle permet à un "peuple" du Cameroun d'affirmer son identité ", m'ont également inspiré dans mon travail sur l'affirmation identitaire des Sawa au Canada. Elle explique dans sa thèse comment la légitimité du Ngondo actuel repose aujourd'hui sur les seuls aspects culturels, d'autres aspects, tels que le pouvoir politique, étant amoindris. Les Sawa sont identifiables par leurs traditions, qui perdurent à travers leur histoire et donnent une véritable signification au terme de culture vivante. L'auteure s'est interrogée sur une éventuelle invention de la tradition et après réflexion, elle suggère la conception de « réinvention » (Dessajan 2010). Je m'interroge effectivement sur cette conception de réinvention du Ngondo dans cet article.

Au regard de l'attachement des Sawa à leur tradition et à leur culture, le maintien des liens avec leur terre d'origine apparaît indispensable quel que soit le lieu géographique où ils se trouvent, ceci dans le but de perpétuer leur tradition et sans doute également de s'identifier aux autres communautés dans un espace multiethnique. Ainsi, la diaspora sawa entend y parvenir en créant des représentations du Ngondo dans différents pays. C'est dans 
cette perspective qu'on assiste à la mise sur pied de sections Ngondo en France, aux États-Unis, en Allemagne, en Tunisie, au Canada, etc. Celle du Canada a pris l'initiative d'organiser depuis 2007 une fête qui, allant de la fête Kaba Ngondo en passant par le Ngondo ô Canada 2009, a pu donner progressivement ce qu'on appelle aujourd'hui le festival Ngondo Afro-Monde de Montréal.

Ce dernier se déroule dans le cadre des "Week-end du monde $»^{5}$ au parc Jean Drapeau de Montréal, le lieu où l'on assiste à une véritable mise en scène des expressions culturelles produites par une multitude de festivals distincts, originaires d'une soixantaine de pays. Il représente la culture africaine à ce grand rendez-vous culturel, et dès lors le problème de recherche surgit, celui de l'expression et de la mise en valeur de la culture des Sawa. Comment interpréter le choix de ce très grand événement culturel où s'entremêlent une variété d'expressions culturelles ? Comment le festival Ngondo Afro-Monde peut-il permettre aux Sawa de s'adapter, et leur être utile dans le contexte d'un pays d'immigration et étranger ? Est-ce que ce festival veut tout simplement se différencier des autres formes de mise en valeur du Ngondo ? Est-il possible de procéder à la mise en valeur d'une culture en faisant la promotion de l'interaction et des échanges entre les cultures sans compromettre, voire sans transformer, les valeurs intrinsèques de celle-ci ? La mise en valeur du Ngondo prônée par le festival Ngondo Afro-Monde n'est-elle pas une forme de folklorisation de la fête en vue de répondre à de nouvelles affirmations identitaires?

Pour mieux trouver des réponses à ces questions, il s'agira de faire une analyse comparée de la fête du Ngondo à Montréal et de celle de Douala. Je me dois d'expliquer la grille analytique dans laquelle on pourra lire clairement les différences et les points communs des rubriques qui les constituent. C'est-à-dire que le lecteur saura si les lieux, les costumes, la gastronomie, les rituels, les participants et les jeux sont les mêmes ou non, dans la pratique de cette fête au Cameroun et au Canada. Il sera question bien évidemment de montrer la transformation et l'adaptation de celle-

5. L'événement « Week-ends du monde » du parc Jean Drapeau offre aux Montréalais de toutes origines une vitrine permettant de célébrer et de partager leur culture et leurs traditions grâce à la musique, la danse et la cuisine. Depuis 2005, cet événement présenté par Loto-Québec connaît un essor considérable auprès de la population montréalaise curieuse de connaître toute la richesse culturelle des diverses communautés venues s'établir à Montréal ; près de 200000 personnes ont assisté à l'événement en 2012 (voir le site Internet du parc Jean-Drapeau, http://www.parcjeandrapeau.com/evenements/week-ends-du-monde/, consulté le 18-03-2013). 
ci à travers précisément l'environnement climatique. Je montrerai à ce niveau comment le climat très différent de celui du Cameroun impose une adaptation de la fête plutôt en été (juillet) qu'en hiver (décembre) comme c'est le cas au Cameroun. J'évoquerai également ce que représente cette fête pour des Sawa du Canada, quelle est leur pensée vis-à-vis du symbolisme de certains rituels et pratiques représentés à la fête au Canada dans le souci de valoriser la culture sawa.

\section{Les points communs ou les similitudes}

\section{La nourriture}

Pendant les fêtes du Ngondo au Canada, depuis l'événement Ngondo ô Canada 2009 au festival Ngondo Afro-Monde, les aliments présentés et consommés sont les mêmes qu'au Cameroun. Toutes les personnes interrogées dans le cadre de mon travail de terrain me l'ont confirmé, et j'en ai eu moi-même la confirmation en participant à certaines activités du festival pour l'édition 2012. J'ai été surpris de constater qu'à Montréal on peut se procurer toutes les épices dont on a besoin pour des mets sawa et africains en général, l'essentiel étant d'avoir suffisamment d'argent, compte tenu du coût du transport. Par ailleurs, on peut même trouver des feuilles de bananier que l'on utilise généralement pour emballer certains mets spéciaux tels que les gâteaux traditionnels (le koki, le gâteau de pistache, etc.). On trouve sur place à Montréal la quasi-totalité des ingrédients entrant dans la composition de ces mets. Depuis une vingtaine d'années, l'ouverture progressive d'épiceries africaines a permis le développement de la consommation d'aliments camerounais.

La possibilité de s'approvisionner à Montréal en aliments et ingrédients de leur cuisine fait que les Sawa parviennent à manger et à présenter les mêmes nourritures pendant leurs cérémonies festives. Ils profitent aussi du statut cosmopolite de la ville de Montréal, qui a une politique culturelle très favorable à l'expansion des cultures, à la promotion de la diversité culturelle. Bon nombre d'ingrédients se retrouvent aussi chez d'autres peuples d'Afrique, d'Asie et d'Amérique Latine. Tous ces peuples sont représentés à Montréal et possèdent eux aussi leurs propres épiceries où les Sawa peuvent se rendre de temps en temps. On peut citer à cet effet la banane plantain avec laquelle les Sawa font le mussolè (plantain frit), et qui est du même type que celle du Mexique ou du Brésil ; ou bien le ndjansan, épice qu'emploient les Sawa pour la marinade du poisson et du poulet et qui est aussi utilisée par les peuples ashanti du Ghana, mais sous un autre 
nom, le plus souvent pour assaisonner des sauces d'arachides grillées ${ }^{6}$.

Il est important de souligner que Montréal est la ville qui regroupe le plus grand nombre des immigrants Camerounais du Canada, parmi lesquels figurent des commerçants dont la majorité travaillent dans l'import-export. Ils approvisionnent le marché Montréalais en ingrédients et produits exotiques, ce qui permet aux Sawa de confectionner les mêmes plats qu'en Afrique lors de leurs manifestations festives. Paul Ékollo, l'un de mes informateurs lors de notre entrevue en juillet 2012 à Montréal, affirmait que depuis 2009 qu'il est à Montréal, il trouve tout ce dont il a besoin pour préparer ses repas. «Depuis mon arrivée ici, je n'ai jamais cherché à manger une nourriture typiquement sawa sans le pouvoir »; à Montréal, à tout coin de rue, il existe une épicerie africaine dans laquelle on peut se ravitailler.

En outre, la politique culturelle de la ville de Montréal accorde une large visibilité aux expressions culturelles des communautés ; cette politique laisse éclore des événements culturels dont la plupart sont consacrés aux arts culinaires, aux dégustations alimentaires, en l'occurrence lors des Week-ends du monde, des Fêtes gourmandes, du Festi Africa, etc. Ce sont de véritables lieux d'échanges culturels qui permettent aux communautés de découvrir des recettes et des suppléments d'ingrédients entrant dans la composition de certains mets. De ce fait, les Sawa parviennent évidemment à maintenir les mêmes goûts que ceux du Cameroun. Il en est de même dans l'industrie vestimentaire.

\section{Les costumes}

Les événements culturels des Sawa sont réputés par l'exhibition de leur art vestimentaire; ils accordent une attention particulière à la manière de se vêtir pendant ces événements. Au Cameroun comme partout ailleurs, les Sawa sont très vite reconnus à leur accoutrement pendant leurs festivités, par exemple par le pagne officiel du Ngondo, avec, sur fond bleu, des photos, des insignes ou des dessins de différents objets ethnographiques utilisés au quotidien, par le Kaba Ngondo pour les femmes et le pagne velouté (tissus en soie) avec une chemise pour les hommes. Généralement, cette chemise est à manches longues et les hommes sont en plus coiffés d'un chapeau et munis d'un chasse-mouche.

Le Kaba Ngondo est une large robe à manches longues descendant

6. Témoignage de Gislain Walli Koff, jeune homme ghanéen rencontré pendant l'achalandage au stand de la Sawayenne le deuxième jour du festival Ngondo Afro-Monde, le 08 juillet 2012 à Montréal. 
jusqu'aux pieds. Dans Le Paradis tabou, Valère Épée décrit les différents types de kaba, à savoir : le kaba de cérémonie ou mindènè, le kaba élégant ou mukuku et le kaba de maison ou misodi. L'ensemble complet a emprunté des accessoires à d'autres cultures : l'ébasi (modèle) européen, le jupon togolais et l'écharpe igbo du Nigeria, jetée avec élégance sur l'épaule gauche. Depuis la naissance du Ngondo jusqu'à nos jours, le kaba est non seulement la tenue officielle et traditionnelle du Ngondo (d'où le nom de kaba ngondo), mais il est également devenu un costume national féminin, porté lors des cérémonies de toutes sortes. Pour la Journée des femmes le 8 mars comme pour la Fête des mères célébrée au mois de mai, le port du kaba est de rigueur. L'habillement des hommes pendant les fêtes, comme nous l'avons mentionné, représente pour ces messieurs une forme d'affirmation identitaire quel que soit le lieu ou l'occasion. Ils arborent ces vêtements traditionnels lors des fêtes, des célébrations culturelles et même pendant des journées spéciales comme des anniversaires ou des pique-nique localement appelés « barbecues » organisés en été.

Pendant la célébration de 2009, la quasi-totalité des Sawa étaient habillés en tenue traditionnelle, tant les hommes que les femmes. C'était le moment idoine, lors de la présence de leur chef supérieur, de montrer aux Montréalais comment ils s'habillent pendant leurs fêtes. L'accès facile aux tissus, grâce au grand nombre de commerces exotiques de la ville, outre la présence de nombreux stylistes modélistes africains et même sawa, rendent aisée la confection de ces costumes appropriés aux événements culturels comme le veut la tradition pendant les fêtes du Ngondo. Elsa Benji, artiste musicienne au festival Ngondo 2012, atteste qu'elle procure toutes les qualités de tissu pour les costumes sawa, en soulignant qu'il est plus facile de se faire confectionner un costume traditionnel africain à Montréal que partout ailleurs au Canada.

\section{Les chants et les danses}

Les chants et les danses sont communs à ces deux milieux; en effet, les Sawa que j'ai interrogés et qui avaient déjà participé à une fête du Ngondo au Cameroun et au Ngondo ô Canada 2009, m’ont affirmé qu'on chantait au bord du fleuve Saint-Laurent les mêmes chants qu'au Cameroun ; mais ils étaient par contre très conscients que ces chants n'avaient aucunement pour intention d'invoquer les esprits de l'eau comme au Cameroun. Ils avaient tout simplement pour finalité de se remémorer des événements hautement historiques et significatifs pour leur épanouissement moral, ainsi que de montrer au public non sawa l'interaction qui existe entre les Sawa 
et les cours d'eau. Certaines chansons et danses reviennent plus souvent lors de ces cérémonies du Ngondo, par exemple le sékelè, ou le bolobo; le grand musicien camerounais Manu Dibango a créé un bolobo très célèbre et populaire, "Amouna cassala fam », également chanté par Paul Ékollo officiant au vieux port de Montréal.

Pour ce qui est des danses, l'ambass bey ou le makossa ont toujours fait partie des types de danse ou des rythmes sur lesquels plusieurs candidats entrent en lice dans le cadre des concours de danse. Au Canada, seuls certains jeunes étaient très actifs, tandis qu'au Cameroun, toutes les couches sociales participent aux jeux. Je reviendrai sur cet aspect en abordant les éléments qui font la différence entre le Ngondo du Cameroun et celui du Canada.

\section{Les différences}

Les rituels

La différence fondamentale que l'on peut constater entre la fête du Ngondo à Douala et celle de Montréal se trouve dans la pratique des rituels. $\mathrm{Si}$, au Cameroun, le Ngondo est l'occasion de multiples rituels, allant des petits principes protocolaires jusqu'à l'immersion du vase sacré, au Canada, il est tout à fait différent ; il se caractérise plutôt par des représentations symboliques, dans le but de mettre en valeur la culture sawa dans ce pays d'accueil.

Le rituel d'Essa, pour lequel Paul Ékollo était l'officiant lors du Ngondo ô Canada 2009, n'est qu'un simulacre de ce qui se passe au Cameroun, bien que cela se soit passé au bord de l'eau. Ce rituel est un culte ou une cérémonie cultuelle de conjuration du mauvais sort, qui se déroule généralement lors d'une neuvaine, soit lors de soins intensifs de guérison chez un ganga ou médecin traditionnel, soit en cas de pénurie alimentaire, de catastrophe naturelle ou d'épidémie. Paul Ékollo souligne même que la pandémie du Sida aurait fait autrefois l'objet d'une convocation d'un Essa géant en vue d'implorer l'intervention des ancêtres face à ce fléau qui décime les populations ${ }^{7}$. L'Essa est donc le seul rituel qui a été pratiqué au Canada pendant le Ngondo 2009, et Cyrille Ékwalla l'a qualifié de " pâle copie » ${ }^{8}$ de ce qui se pratique au Cameroun. Cependant, il est important de noter qu'à chaque fois que les Sawa envisagent de faire quelque chose de grand,

7. Entretien avec Paul Ékollo le 29 juillet 2012 à Montréal.

8. Entretien avec C. Ekwalla le 4 septembre 2012 à Montréal. 
ils l'appellent Ngondo : «ce Ngondo, précise Paul Ékollo, ne s'assimile pas au Ngondo du Cameroun ». Herbert Éyoum renchérit en soulignant que celui qui veut vivre le Ngondo des rituels, le Ngondo mystique, doit aller le vivre au Cameroun, car ce qui se fait au Canada n'est qu'une représentation pure et simple, très différente du Ngondo du Cameroun.

On ne peut parler d'une célébration du Ngondo sans parler du rituel d'immersion du vase sacré qui a été évoqué plus haut. Mais il n'en est pas question dans le Ngondo pratiqué hors de son contexte d'origine. Le Ngondo, à la base, est considéré aussi comme un sanctuaire, or on ne peut déplacer un sanctuaire, quelle qu'en soit la raison. En langue duala, massosso ma nyambé veut dire littéralement " les trois piliers d'un foyer ». Ces trois piliers représentent les Douala, les Bassa et les Bakoko (Ngametche 2013). Le foyer de fondation du Ngondo est donc fixé à Douala, en un lieu bien précis, qui ne pourra jamais au grand jamais se déplacer. C'est là le siège du Ngondo, le lieu où les messages des ancêtres sont transférés aux populations par l'entremise des initiés.

\section{Les jeux et concours}

Depuis la relance du Ngondo en 1991, plusieurs jeux et concours ont été intégrés dans la programmation des festivités. Ils constituent la rubrique d'activités la plus flexible de la célébration. Les principaux jeux et concours qui se font au Cameroun, à savoir la course de pirogues, la lutte traditionnelle et le concours Miss Ngondo, n'ont pas eu lieu au Canada. On a plutôt connu des transformations ou des adaptations de ceux-ci, que nous verrons plus bas dans la partie consacrée aux transformations de la fête. La liste des jeux et concours aux festivités du Ngondo est très souvent choisie par les organisateurs en fonction de la capacité de ceux-ci d'attirer le plus de spectateurs. À Montréal en 2009, les jeunes Sawa avaient opté pour le football, à New-York aux États-Unis, l'une des célébrations des fêtes culturelles sawa appelée naturellement « Ngondo aux USA » a connu le volleyball et le basketball. On constate beaucoup de différences en fonction du milieu dans la rubrique des jeux et concours.

Cela peut s'expliquer par la variation du climat et du public : les organisateurs adoptent les jeux qu'ils jugent populaires selon le milieu où ils se trouvent. Cela peut également s'expliquer par le fait que les principaux jeux qui renvoient à l'identité même du Ngondo, comme la course de pirogues, ne peuvent se faire nulle part ailleurs en dehors de Douala, parce qu'il semblerait que cette course ait aussi un caractère sacré et mystique. 


\section{Les manières de penser des participants}

Les participants à la fête du Ngondo au Cameroun ont généralement une pensée beaucoup plus basée sur la sacralité, sur les modes de pensée traditionnelle, alors qu'au Canada, les participants issus de diverses origines ont une manière de penser généralement portée sur des valeurs universelles de représentation. Au Canada, hormis certains Sawa, les participants se préoccupent davantage, par exemple, de comprendre comment les peuples sawa manifestent concrètement la solidarité, comment ils expriment l'amour du prochain, comment ils entendent promouvoir la vérité, la justice et l'entente mutuelle au sein des communautés vivant ensemble. Séverine Dessajan souligne avec beaucoup de détails que toutes ces valeurs universelles s'expriment entre autres dans des activités entendues comme des animations culturelles (Dessajan 2010). Les rythmes de danses comme l'esséwè, le bolobo ou encore l'ambass-bey renvoient aux mouvements d'ensemble qui favorisent la socialisation et qui peuvent s'interpréter comme une invitation à la réconciliation et au pardon.

Au Cameroun, le pouvoir sacré des Sawa existe dans une continuité, dans un renvoi perpétuel aux ancêtres et dans l'existence d'un Dieu suprême, Nyambe. Dans les textes écrits comme dans la tradition orale, le Ngondo se révèle à travers des rituels d'échanges avec les ancêtres et leurs intermédiaires, les miengu, les esprits de l'eau. Dans la vie sociale sawa, la prépondérance d'une force dite vitale revient sans cesse, ce qui conditionne en quelque sorte la manière de penser. Pour sa survie, cette société renvoie aux éléments sociaux qui déterminent l'être humain, c'est-à-dire l'amour, la fécondité, la maladie et la mort. C'est la raison pour laquelle de nombreux rituels se constituent autour de ces éléments. Cette manière de penser passe par la référence aux ancêtres - phénomène que l'on retrouve dans de nombreux groupes bantu (Obenga 2006). Plusieurs histoires légendaires sont consacrées à l'ancêtre mythique des peuples sawa. Une volonté fédératrice émane de cet ancêtre fondateur, comme si tous avaient le même géniteur. C'est autour de lui que se concrétise le souhait de réunir le plus grand nombre de personnes autour de son culte et parallèlement au Ngondo.

Chez les Sawa, le sacré fait de nombreuses références à l'eau, à la mer, aux rivières, soit aux esprits de l'eau, aux poissons, aux crevettes, mais aussi aux animaux qui ont fait ou font encore partie de leur quotidien, comme l'éléphant, la chèvre... Il est vrai que de nombreuses populations côtières qui sont en contact avec le milieu aquatique connaissent les mêmes manifestations d'esprits de l'eau. Le rôle économique de la pêche fut l'élément fondateur de ces croyances. Les esprits de l'eau sont 
omniprésents dans cette société dont les membres étaient, rappelons-le, d'anciens pêcheurs. Les miengu sont à étudier également sous la forme d'une société secrète, la société jengu (Rosny 1987). Toute cette manière de penser, toute cette philosophie des Sawa au Cameroun vis-à-vis du Ngondo est absolument différente de celle des Sawa du Canada qui participent régulièrement à la fête, déjà du simple fait que la fête du Ngondo au Canada ne met aucun accent sur des rituels, sur des principes traditionnels qui constituent l'ensemble de leur mode de vie. Sous d'autres cieux et sous des systèmes politiques différents de celui du Cameroun, le vécu quotidien des Sawa n'est pas forcément conditionné par la pratique du Ngondo. Contrairement à ce qui se passe au Cameroun, les participants à la fête du Ngondo au Canada et même ailleurs en Occident sont enrôlés dans des sociétés modernes fortement industrialisées, où les rapports sociaux sont davantage rythmés par les normes capitalistes. Les gens sont convaincus que c'est grâce à leur travail et leur dynamisme qu'ils vivent, qu'ils parviennent à résoudre leurs problèmes. Or, au Cameroun, bien des participants à la fête du Ngondo sont de leur côté convaincus que c'est grâce à la magnanimité de leurs ancêtres, à la croyance aux cultes et à beaucoup de rituels exécutés dans leur environnement qu'ils trouvent des solutions à tous leurs problèmes. Du coup, au Cameroun, la fête du Ngondo n'est pas qu'un simple divertissement, un moyen parmi tant d'autres de renforcer les liens sociaux, de rencontrer d'anciens amis, de prendre un verre en commun, mais plutôt un élément incontournable et même primordial pour beaucoup de Sawa.

\section{Les transformations et les adaptations}

\section{La période de la fête}

La période de la fête du Ngondo n'a pas été choisie au hasard ; elle a évolué depuis l'époque précoloniale jusqu'à l'époque postcoloniale, bien que les avis restent partagés sur ce point. Léopold Moumé Etia souligne qu'avant l'arrivée des colons, la fête du Ngondo s'organisait au moment des grandes marées de juin, période où les vagues repoussent jusqu'au niveau des côtes certains fruits de mer très prisés par les Sawa dans leur alimentation . De nos jours, elle se célèbre la première semaine du mois de décembre, représentant la fin de l'année, qui est un moment propice pour rendre grâce aux dieux par l'intermédiaire des ancêtres et d'obtenir d'eux des prédictions pour l'année à venir (Moumé-Étia 1981 : 32). 
À Montréal, la fête ne pouvant pas se célébrer en décembre ${ }^{9}$ comme à Douala, à cause du climat peu favorable aux activités de plein air, les Sawa ont adapté la fête à la période de l'été, moment qui leur est favorable. Le mois d'août est le mois qui a vu le premier Ngondo naître au Canada. Il faut remarquer que tous les événements culturels des Sawa au Canada s'organisent toujours en été, c'est-à-dire entre le mois de juin et le mois d'août. À part des raisons climatiques, on note également que c'est la période pendant laquelle beaucoup de travailleurs prennent leur congé annuel et que c'est aussi le moment qui correspond le plus souvent aux vacances scolaires, ce qui permet aux enfants de participer aux activités.

\section{Simulation de la course de pirogues}

La course de pirogues est une activité majeure du Ngondo ; elle a été introduite dans la programmation de la fête depuis les années 1990. Cette course et la lutte traditionnelle sont devenues les activités phares du Ngondo en ce qui concerne le volet sport. La pirogue en elle-même représente un symbole très important dans la vie des Sawa. Étant les peuples de l'eau, tels qu'ils aiment à se définir, les Sawa ne pourraient mener aucune activité sans pirogue : elle est en même temps un moyen de transport d'une région à l'autre par voie maritime, un outil de travail pour les pêcheurs et pour ceux qui extraient du sable des fond des eaux, enfin elle est un sport. La pirogue fait partie de la vie des Sawa.

Pour toutes ces raisons, à la place de la course de pirogues telle qu'elle se tient au Cameroun, la fête du Ngondo ô Canada 2009 a connu une simulation de cette course en plein air, dans le simple souci de représenter matériellement ce qu'on entend par course de pirogues, ne pouvant pas aller dans le fleuve Saint-Laurent puisque n'ayant aucun lien historique avec celui-ci. Les jeunes de l'association Jeunesse Sawa ont eu l'ingénieuse idée de symboliser la course de pirogues à travers une magnifique création. En ajustant des pagnes sur une longueur d'environ 10 mètres, et en les arrangeant sous une forme ovale, ils ont fabriqué " une pirogue » dans laquelle une dizaine de jeunes hommes munis chacun d'une pagaie marchaient en simulant l'action de pagayer. Tout au long de la marche, un autre jeune se faisait le plaisir d'expliquer cette course de pirogues symbolique qui a un sens extrêmement profond chez les Sawa (voir Figure

9. Le mois de décembre étant le début de l'hiver au Canada, la température à l'extérieur varie entre $-25^{\circ}$ et $-10^{\circ} \mathrm{C}$, tandis qu'à Douala à la même période elle se situe entre 25 et $30^{\circ} \mathrm{C}$. Ce n'est qu'en été que la température dépasse les $20^{\circ}$ à Montréal, ce qui permet donc les activités de plein air. 
1). Cela représentait une mise en valeur de la culture des Sawa et de leur mode de vie dans lequel l'eau et la pirogue constituent des référents culturels extrêmement importants.

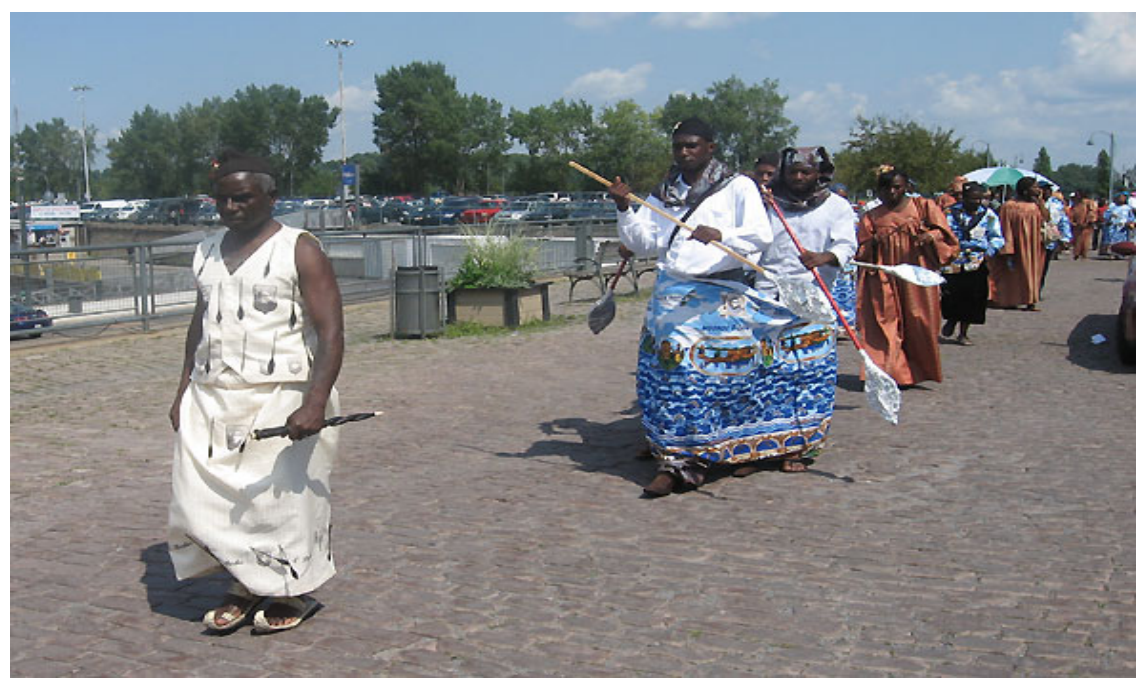

Figure 1. Hommes et femmes sawa en tenue traditionnelle; les hommes sont munis de pagaies et simulent la course de pirogue symbolique (Photo Circom 2009).

Dénomination de la fête après 2009 et conception du projet "Leaders et personnalités noires " inspiré du concours Miss Ngondo

Après la fête du Ngondo ô Canada 2009, Solidarité Sawa se renforce ; on assiste à Ottawa à la création de Ngonda'a Sawa, une association qui vise non seulement à maintenir le lien avec le pays d'origine, mais aussi à rassembler les Sawa du Canada et à promouvoir la culture sawa dans sa globalité.

Solidarité Sawa quant à elle explore d'autres horizons en mettant sur pied le festival Ngondo Afro-Monde, qui valorise la gastronomie sawa dans un très grand événement culturel (Week-ends du monde du parc Jean Drapeau) où sont représentés une soixantaine de pays qui mettent en scène leurs différentes expressions culturelles sur tous les plans. Le festival Ngondo Afro-Monde y est la seule représentation culturelle originaire d'Afrique subsaharienne. Il fait ainsi en même temps la fierté des Camerounais et des Africains, en devenant une fête qui permet d'identifier les Africains en général et les Camerounais en particulier, et plus précisément les Sawa. 
Sous cet angle, la fête du Ngondo joue un rôle identitaire afro-centriste au sein du foisonnement des expressions culturelles représentées au parc Jean Drapeau de Montréal, d'où sa dénomination Festival Ngondo Afro-Monde. Plusieurs fois lors de nos multiples entretiens à Montréal après le festival (juillet 2012), et très récemment encore au téléphone, Agnès Eyoum n’a cessé de me rappeler l'objectif majeur de ce festival qui est de fédérer dans la mesure du possible toutes les forces vives de la diaspora africaine pour la promotion globale et totale de la culture africaine. C'est de là qu'est née sans doute la vision afro-descendante de la section jeune de Solidarité Sawa, Jeunesse Sawa. Dans la même perspective, l'on peut dire que le concours Miss Ngondo a inspiré le concours « Leaders et personnalités noires » qu'organise Solidarité Sawa dans le cadre de ses activités annuelles. Tout comme Miss Ngondo au Cameroun, ce concours ne valorise pas seulement la beauté physique de ses candidats et ses candidates, mais aussi et surtout leur beauté morale, leurs valeurs intrinsèques en vue d'en faire des personnalités de référence dans leur société. On remarque à ce niveau comment le Ngondo prend une autre tournure, avec un sens différent de ce qu'on connaît à la base, c'est-à-dire au Cameroun, différent également de ce qui s'est passé en 2009 sous la coordination du président du Ngondo venu pour la circonstance.

\section{La fête du Ngondo au Canada comme folklore ?}

La question de savoir si le Ngondo au Canada ne se réduisait pas à une grande manifestation folklorique me paraît jusque-là importante malgré les analyses qui précèdent. Comme l'écrit Nicole Belmont dans sa définition du folklore, ce concept serait apparu au début du XVIII ${ }^{\mathrm{e}}$ siècle avec la fondation de l'Académie celtique en 1805. Ce fut l'occasion de collecter pour la première fois les traditions dites populaires, mais ceci est à replacer dans un contexte de nationalisme. Par la suite, ce concept a regroupé des pratiques plutôt en voie de disparition dans une civilisation en pleine gestation, en pleine mouvance, avec l'apparition d'une culture dite vivante. En outre, «si le terme a disparu de l'usage scientifique, il n'en reste pas moins qu'il y a production de folklore dès que deux cultures, l'une dominante, l'autre dominée, coexistent l'une avec l'autre » (Belmont 1992 : 283-284).

Dans le cadre du Ngondo au Canada, on peut en effet parler de plusieurs cultures qui sont en interaction dans une perspective de multiculturalisme où les cultures minoritaires sont dominées. Aujourd'hui, à travers leurs manifestations culturelles qui s'expriment par la résurgence d'un certain folklore, elles mettent de l'avant leur position de « minorités ethniques », 
dans un but de consistance identitaire par rapport aux autres cultures représentées à Montréal. L'un de mes informateurs me faisait remarquer qu'une culture ne vit que lorsqu'elle se manifeste, et la manifestation de la culture Sawa passe aujourd'hui par des éléments tels que le chant, la danse, le port du vêtement traditionnel. Ce sont là des témoignages d'une culture dite vivante.

Même si certains peuvent voir à travers toutes ces représentations culturelles sawa une manière folklorique d'affirmer leur identité, la majorité des Sawa au Canada n'admettent pas l'idée d'une folklorisation de leurs diverses manifestions. Ils trouvent que se replier sur la tradition ancestrale est un acte indispensable pour le plein épanouissement de l'individu, l'important ici étant d'éviter toute forme de communautarisme à outrance qui embrigaderait la créativité et condamnerait toute initiative de modernité ou de mondialisation. Car le repli identitaire à travers des manifestations festives est aussi l'un des moyens les plus sûrs de perpétuer et de garder ses repères.

Certains membres de l'élite sawa émettent des réserves sur la tradition en général, et pensent que le Ngondo, que ce soit celui du Canada ou celui du Cameroun, n'est de nos jours qu'une forme de réminiscence du passé n'ayant plus rien à voir avec «l'expression symbolique initiale ». Tout l'aspect du signifiant ancestral a, selon eux, disparu. Ils soutiennent que les jeunes, avant de se réapproprier des traditions dites ancestrales, auraient déjà dû essayer de les connaître, de les maîtriser et surtout de les comprendre : «Répéter ce qu'on a vu faire ne sert à rien si on n'essaye pas de comprendre les actes. C'est faire une sorte de folklore à la bretonne ou de toute autre région » (Dessajan 2010 : 289).

Cet article permet de voir clairement ce qui fait la différence entre la fête du Ngondo au Cameroun et au Canada. Les nombreux rituels et le sens sacré prédominant qui caractérisent la fête au Cameroun ne sont pas du tout ce qui se vit dans la fête du Canada ; lorsque cette dernière a débuté, en 2009, on avait assisté à un simulacre des rituels de conjuration du mauvais sort appelé l'Essa, suivi de quelques représentations culturelles et de la valorisation de la gastronomie sawa. Mais après 2009, le Ngondo au Canada est devenu festival Ngondo Afro-Monde, basé sur la gastronomie sawa représentant la culture africaine en général au grand événement des Week-ends du monde du parc Jean Drapeau de Montréal.

Mon implication personnelle en tant que bénévole à l'édition 2012 de ce festival m'a permis de voir effectivement de près comment se passe 
cette fête. Par conséquent, je me suis rendu compte que la fête du Ngondo telle qu'elle se pratique au Canada n'a rien à voir avec les rituels et le côté sacré qu'on lui connaît au Cameroun. C'est plutôt une manière de faire connaître la culture sawa au-delà des frontières camerounaise ; c'est un volet de la mondialisation dont les communautés culturelles devraient se servir, c'est-à-dire profiter d'un espace de visibilité à l'international pour présenter les spécificités culturelles d'une communauté. C'est également une forme de mise valeur qui permet aux cultures de s'exprimer et de vivre sans forcément verser dans la folklorisation.

\section{Grilles analytiques}

\begin{tabular}{|c|c|c|}
\hline & $\begin{array}{c}\text { Fête du Ngondo au } \\
\text { Canada }\end{array}$ & $\begin{array}{c}\text { Fête du Ngondo au } \\
\text { Cameroun }\end{array}$ \\
\hline Lieu & $\begin{array}{l}\text { Plein air, vieux port de Montréal } \\
\text { (2009); parc Jean Drapeau } \\
\text { (depuis 2010) }\end{array}$ & $\begin{array}{l}\text { Plein air, rives du fleuve Wouri, } \\
\text { considéré comme le lieu sacré } \\
\text { des célébrations des Sawa, } \\
\text { (processions, incantations, } \\
\text { initiations, actions de grâce, etc. }\end{array}$ \\
\hline Période & Été (de juin à août) & $\begin{array}{l}\text { Hiver (première semaine de } \\
\text { décembre) }\end{array}$ \\
\hline Costumes & $\begin{array}{l}\text { Femmes: Kaba Ngondo, foulard } \\
\text { Hommes: pagne attaché autour } \\
\text { des reins, chemise à manches } \\
\text { longues, chasse-mouche } \\
\text { (facultatif) }\end{array}$ & $\begin{array}{l}\text { Femmes: Kaba Ngondo, foulard } \\
\text { Hommes : pagne attaché autour } \\
\text { des reins, chemise à manches } \\
\text { longues, chasse-mouche } \\
\text { (facultatif), beaucoup d'attributs } \\
\text { divers des notables et chefs } \\
\text { supérieurs }\end{array}$ \\
\hline Gastronomie & $\begin{array}{l}\text { Principaux plats: poisson braisé, } \\
\text { missolè (plantain frit), poulet } \\
\text { braisé, mbéatowè (crevettes } \\
\text { sautées), riz sauce oignon, } \\
\text { beignets de farine de blé } \\
\text { Breuvages : vin, bière, coca, } \\
\text { punch de gingembre, jus } \\
\text { d'ananas, rhum }\end{array}$ & $\begin{array}{l}\text { Principaux plats: poisson braisé, } \\
\text { missolè (plantain frit), poulet } \\
\text { braisé, mbéatowè (crevettes } \\
\text { sautées), riz sauce oignon, } \\
\text { beignets de farine de blé, Ndolè, } \\
\text { miondo, etc. } \\
\text { Breuvages : vin de palme (sève } \\
\text { blanche du palmier à huile, } \\
\text { boisson très prisée lors des } \\
\text { cérémonies traditionnelles), } \\
\text { bière, coca, punch de gingembre, } \\
\text { jus d'ananas, rhum }\end{array}$ \\
\hline Chants et danses & $\begin{array}{l}\text { Rythmes d'esséwè : Assiko, } \\
\text { Bolobo }\end{array}$ & $\begin{array}{l}\text { Rythmes d'esséwè : Asiko, } \\
\text { Bolobo }\end{array}$ \\
\hline
\end{tabular}




\begin{tabular}{|c|c|c|}
\hline Participants & $\begin{array}{l}\text { Public très diversifié avec la } \\
\text { présence de plus de } 80 \% \text { des } \\
\text { Sawa du Canada pour l'édition } \\
2009\end{array}$ & $\begin{array}{l}\text { Public à forte concentration } \\
\text { camerounaise en générale et } \\
\text { sawa en particulier }\end{array}$ \\
\hline Jeux & $\begin{array}{l}\text { Pas de jeux comme au } \\
\text { Cameroun, cependant un } \\
\text { match du football avait été } \\
\text { organisé par des jeunes en } 2009\end{array}$ & $\begin{array}{l}\text { Principaux jeux et concours : } \\
\text { Course de pirogues } \\
\text { Concours Miss Ngondo } \\
\text { Lutte traditionnelle } \\
\text { Jeux et concours additionnels : } \\
\text { Course de cyclisme } \\
\text { Concours de chanson religieuse } \\
\text { Concours de cuisine } \\
\text { Concours de danse }\end{array}$ \\
\hline Rituels & $\begin{array}{l}\text { Aucun rituel, à l'exception de } \\
\text { l'Essa de } 2009 \text { au bord du Saint- } \\
\text { Laurent à Montréal sous forme } \\
\text { de remémoration }\end{array}$ & $\begin{array}{l}\text { Beaucoup de rituels qui } \\
\text { constituent véritablement } \\
\text { le gage de l'alliance avec les } \\
\text { esprits et les ancêtres, avec, } \\
\text { entre autres: } \\
\text { - le dépôt d'une gerbe de fleurs } \\
\text { sur les tombes des héros } \\
\text { - l'immersion du vase sacré dans } \\
\text { le Wouri } \\
\text { - l'interprétation du message } \\
\text { venant des ancêtres } \\
\text { - la cérémonie de conjuration du } \\
\text { mauvais sort (l'Essa) } \\
\text { - les paroles de bénédiction du } \\
\text { président du Ngondo, etc. }\end{array}$ \\
\hline Manière de penser & $\begin{array}{l}\text { Manière de penser basée sur } \\
\text { des valeurs universelles de } \\
\text { représentation surtout pour des } \\
\text { non-Sawa et certains Sawa aussi }\end{array}$ & $\begin{array}{l}\text { Manière de penser centrée sur } \\
\text { la tradition et le sacré, croyance } \\
\text { très forte à la tradition pouvant } \\
\text { friser la dépendance selon des } \\
\text { «vrais Sawa » }\end{array}$ \\
\hline
\end{tabular}

\section{Références}

Belmont Nicole, 1992, «Folklore ». In Pierre Bonte et Michel Izard, dir., Dictionnaire de l'ethnologie et de l'anthropologie : 283-284. Paris, PUF.

Dessajan, Séverine, 2000, «Les Duala et le Ngondo, ou comment une assemblée traditionnelle permet à un peuple du Cameroun d'affirmer son identité », Thèse de doctorat en Anthropologie sociale et ethnologie, 
Université Paris Descartes et École des hautes études en Sciences Sociales.

Doumbe Moulongo, Maurice, 1972, Le Ngondo. Assemblée traditionnelle du peuple duala, Yaoundé, Centre d'Edition et de Production de manuels et d'Auxiliaires de l'Enseignement.

Elamé, Esoh, 2006, « La prise en compte du magico-religieux dans les problématiques du Développement durable, le cas du ngondo chez les peuples sawa du Cameroun ». Vertigo 7(3) : http://vertigo.revues. org/2685

Épée, Valère (Ebele Wei), 1999, Le paradis tabou. Autopsie d'une culture assassinée. Douala, Éditions Cerac.

Guidroux, Linda, 2011, Appartenances culturelles et ethnologie des migrations : les Bretons migrants au Québec depuis 1950. Thèse de doctorat en Ethnologie et patrimoine, Université Laval.

Moumé Étia, Léopold Jengu (Djengou), 1981, Mammy Water. Douala, à compte d'auteur.

Mveng, Engelbert, 1963, Histoire du Cameroun, Paris, Présence Africaine. Obenga, Théophile, dir., 1989, Les Peuples Bantu, migrations, expansion et identité culturelle. Actes du colloque international de Libreville, $1^{\text {er }}-6$ avril 1985. Paris, L'Harmattan.

Ngametche Robinson, 2013, «Le festival Ngondo Afro-Monde de Montréal comme adaptation de l'identité culturelle des Sawa au Canada », Mémoire de Maîtrise en Ethnologie et patrimoine, Université Laval. Rosny, Éric de, 1987, Les yeux de ma chèvre. Paris, Plon. 